Using electrochemistry-total internal reflection imaging ellipsometry to monitor biochemical oxygen demand with polyaniline modified electrode

Jia Shen, Wei Liu, Yu Niu, Meng Li, and Gang Jin

Citation: Journal of Vacuum Science \& Technology B 38, 024001 (2020); doi: 10.1116/1.5129606

View online: https://doi.org/10.1116/1.5129606

View Table of Contents: https://avs.scitation.org/toc/jvb/38/2

Published by the American Vacuum Society

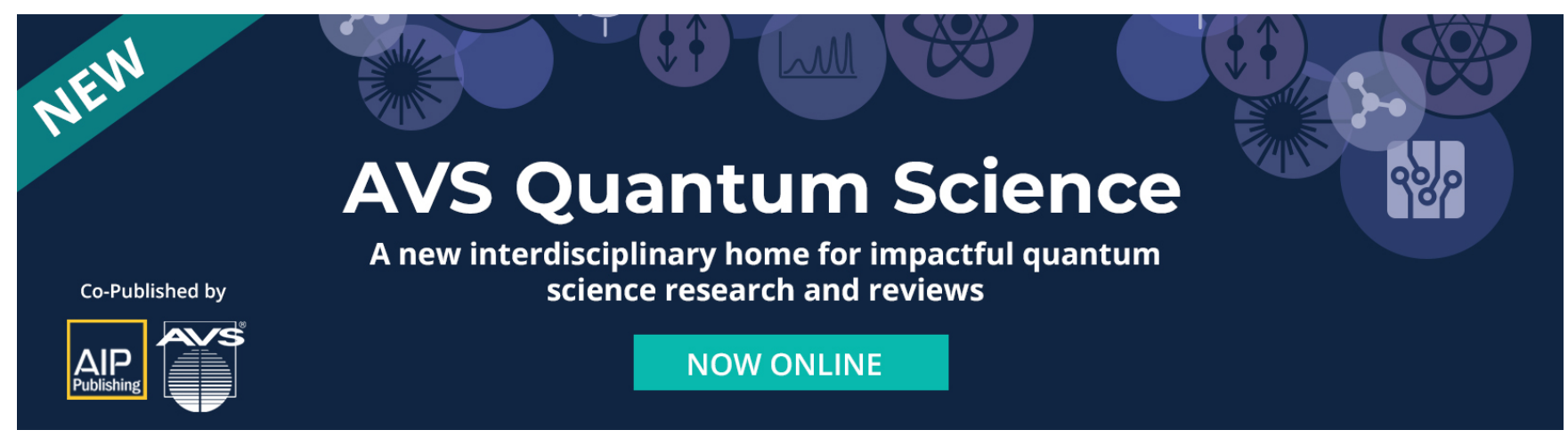




\title{
Using electrochemistry-total internal reflection imaging ellipsometry to monitor biochemical oxygen demand with polyaniline modified electrode
}

Cite as: J. Vac. Sci. Technol. B 38, 024001 (2020); doi: $10.1116 / 1.5129606$

Submitted: 30 September 2019 - Accepted: 3 January 2020 .

Published Online: 27 January 2020
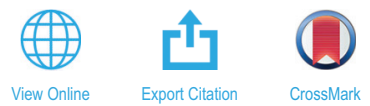

Jia Shen, ${ }^{1,2, a)}$ Wei Liu, , ${ }^{1, a)}$ Yu Niu, ${ }^{7, b)}(1)$ Meng Li, ${ }^{3, c)}$ and Gang Jin $^{1,2}$

\begin{abstract}
AFFILIATIONS
${ }^{1} \mathrm{NML}$, Beijing Key Laboratory of Engineered Construction and Mechanobiology, Institute of Mechanics, Chinese Academy of Sciences, 15 Bei-Si-Huan West, Beijing 100190, China

${ }^{2}$ School of Engineering Science, University of Chinese Academy of Science, 19 Yu-quan Road, Beijing 100049, China

${ }^{3}$ State Key Laboratory of Analytical Chemistry for Life Science, School of Chemistry and Chemical Engineering, Nanjing University, Nanjing 210093, China
\end{abstract}

Note: This paper is part of the Conference Collection: 8th International Conference on Spectroscopic Ellipsometry 2019, ICSE.

${ }^{a}$ Contributions: J. Shen and W. Liu contributed equally to this work.

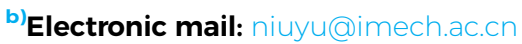

${ }^{c)}$ Electronic mail: limeng@nju.edu.cn

\begin{abstract}
Biochemical oxygen demand (BOD) is an important indicator for characterizing organic pollution in water. So various BOD sensors have been developed for BOD detection. BOD sensors based on a Clark electrode have the problem of signal drift. To overcome this problem, a combined electrochemical and total internal reflection imaging ellipsometry sensor has been proposed. To improve the sensitivity, polyaniline (PAn) and PAn incorporated with Fe(III) meso-tetra (4-sulfonatophenyl) porphyrin (FeTSPP) have been introduced on the working electrode. The results show that the combination system is effective for BOD detection with PAn. By incorporating FeTSPP, the optical and electrochemical responses of a PAn modified gold chip to BOD are improved significantly. Finally, the relationship between the optical and electrochemical signals is investigated by establishing a glass/Au/PAn-based film/liquid model. The analysis reveals that both signals represent the ratio change of the oxidized state of PAn in a similar way.
\end{abstract}

Published under license by AVS. https://doi.org/10.1116/1.5129606

\section{INTRODUCTION}

Biochemical oxygen demand (BOD) is the amount of dissolved oxygen (DO) required by micro-organisms to degrade organic matter in water under certain conditions. BOD has great practical significance in water quality monitoring and evaluation, because it is a comprehensive indicator reflecting the content of organic pollutants in water. The standard method for estimating BOD is the well-known $\mathrm{BOD}_{5}$, which needs five days of incubation at $20^{\circ} \mathrm{C}$ in darkness. ${ }^{1}$ As this method requires not only five days but also complicated procedures and skilled analysts, ${ }^{2}$ studies have been conducted to develop alternative methods for BOD measurement. Various BOD sensors based on DO detection consumed by immobilized micro-organisms have been developed since a BOD sensor that used immobilized micro-organisms with an oxygen electrode was first reported in 1977..$^{3-6}$ These BOD sensors generally use a Clark electrode as a transducer. ${ }^{7}$ The main problem of the BOD biosensor is signal drift due to electrode aging, and it is necessary to clean the electrode and precalibration frequently. ${ }^{8}$

To overcome the signal drift, our previous work has proposed a novel sensor combining electrochemical and total internal reflection imaging ellipsometry (EC-TIRIE) systems. ${ }^{9}$ In the combination system, a gold film is used as both the working electrode and the optical sensing surface, so electrochemical and optical signals can be acquired simultaneously and confirmed. By using only the gold film, the signal-to-noise ratio of the optical signal is insufficient to realize DO quantitative detection. In order to improve the performance of 
the EC-TIRIE sensor, polyaniline (PAn), an oxygen-sensitive polymer with special electrical and optical properties, ${ }^{10}$ is introduced on the sensing surface. There are several methods for immobilization of PAn on the gold electrode, such as cyclic voltammetry (CV), ${ }^{11,12}$ surface graft, ${ }^{13,14}$ and chemically modified electrode through a PAn composite. ${ }^{15}$ Here, a PAn film is immobilized on the gold chip by the CV method. With PAn modification, the optical signal of the gold chip for DO detection is amplified, and the optical and electrochemical signals are in good accordance with each other. To further improve the optical sensitivity, an oxygen-sensitive molecule, Fe(III) meso-tetra (4-sulfonatophenyl) porphyrin (FeTSPP), is incorporated into PAn during the film generation, and both the optical and electrochemical responses to $\mathrm{DO}$ are improved significantly. ${ }^{16}$

In this paper, the PAn-modified EC-TIRIE sensor is used to detect BOD by immobilizing a microbial membrane, and FeTSPP is introduced into the PAn film on the gold chip to further improve the responses of the sensor to BOD. Both the electrochemical and optical responses to different BOD concentrations are studied. The results show that the PAn-FeTSPP-modified electrode has much higher sensitivity to BOD than the PAn-modified electrode. Finally, the relationship between the optical and electrochemical signals of the PAn-based-modified EC-TIRIE sensor is investigated by establishing a glass/Au/PAn-based film/liquid model. The analysis reveals that both the electrode potential variation and the ellipsometric parameters' variation represent the ratio change of the oxidized state of PAn in a similar way.

\section{METHODOLOGY AND EXPERIMENT}

\section{A. Chemicals}

Sulfuric acid (98\%) was purchased from Beijing Chemical Works. Phosphate-buffered saline (PBS) was purchased from Sigma-Aldrich. Aniline (99.7\%) was bought from J\&K Scientific Ltd. FeTSPP was purchased from Frontier Scientific (USA). A standard solution with a BOD value of $20 \mathrm{mgl}^{-1}$ was prepared by National Institute of Metrology, China. Solutions of other BOD values were obtained by dilution of the above solution with PBS buffer. De-ionized water was obtained from the MILLI-Q purification system (18.2 MW at room temperature) and used to prepare all the solutions. Nitrogen (99.9\%) and oxygen (99.9\%) were acquired from Beijing Oxygen Factory. The microbial membrane was purchased from Nissin Electric Co. Ltd.

\section{B. Apparatus}

Figure 1 shows the schematic diagram of the combined electrochemical and TIRIE (EC-TIRIE) setup for BOD detection. TIRIE is imaging ellipsometry operated in the internal reflection mode. ${ }^{17}$ The light beam from the light source passes through a polarizer and a compensator; the polarized beam propagates perpendicularly into the prism and finally onto the gold film. When the incident angle is larger than the critical angle, the total internal reflection phenomenon occurs; at the same time, an evanescent field appears on the gold film surface and is used to monitor the interactions. The optical signal of the polarized light intensity variation is detected by the CCD camera in gray scale format. The image captured by the CCD camera is shown in Fig. 2. After the PAn generation, the PAn film completely covers the region inside the red circle.

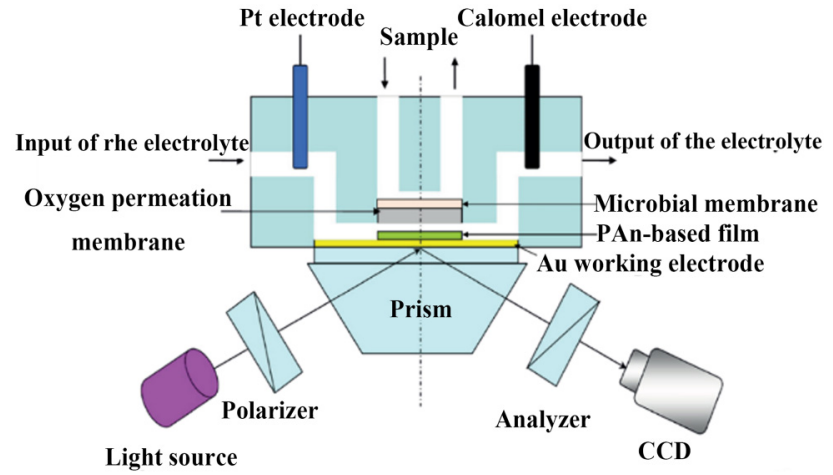

FIG. 1. Schematic illustration of the combined EC-TIRIE system to BOD detection.

Thus, we take the averaged gray scale value of the pixels inside the red circle as the optical signal. The electrochemical experiments are performed using a VersaSTAT3 electrochemical system (Princeton) for controlling the working electrode potential and recording the potential and current. An electrochemical reactor is placed on top of the gold film for holding the tested solution. The electrochemical cell containing the electrolyte is separated from the sample cell by an oxygen permeable membrane. The microbial membrane is attached to the top of the oxygen permeable membrane. Degradation of organic matter in water samples by micro-organisms will consume DO, resulting in a decrease in DO diffusing through the oxygen permeable membrane into the electrolyte. The gold film is used as the working electrode; a wound $\mathrm{Pt}$ wire counter electrode and a saturated calomel reference (SCE) electrode are inserted into the electrochemical cell from the top opening. All the potentials are given with respect to this reference electrode. A commercial BOD biosensor

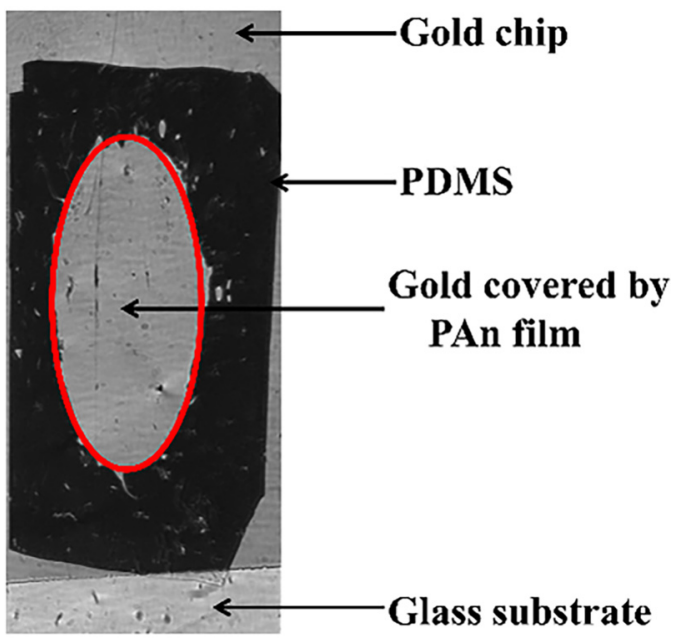

FIG. 2. Image captured by the CCD camera. 
composed of an oxygen electrode and a microbial membrane is used to determine BOD values of BOD standard solutions when compared with the EC-TIRIE sensor.

Atomic force microscopy (AFM): The surface morphology of the PAn film and the PAn-FeTSPP film on the gold substrate was characterized by Multimode AFM Nanoscope IIIa scanning probe microscope (manufactured by Digital Instruments Veeco, USA). All AFM measurements were made in a tapping mode using a silicon carbide tip with a frequency around $300 \mathrm{kHz}$.

\section{Experimental procedure}

The microbial membrane needs to be immersed in a beaker containing $10 \mathrm{mM}$ PBS solution for $72 \mathrm{~h}$ to activate the micro-organisms in the microbial membrane. After the activation, the microbial membrane is fixed in the sample cell and close to the oxygen permeation membrane.

PAn film is grown on the gold substrate from $0.5 \mathrm{M} \mathrm{H}_{2} \mathrm{SO}_{4}$ containing $0.1 \mathrm{M}$ aniline by electrosynthesis. ${ }^{9}$ After the electrosynthesis, $0.1 \mathrm{M} \mathrm{H}_{2} \mathrm{SO}_{4}$ deaerated with nitrogen as a supporting electrolyte is pumped into the electrochemical cell. BOD is measured under the application of a small cathodic current $(0 \mathrm{~mA})$ to the modified gold chip, by passing BOD standard solutions with saturated DO from a high concentration to a low concentration through the sample cell. Degradation of organic matter in water samples by micro-organisms will consume DO, resulting in a decrease in DO diffusing through the oxygen permeable membrane into the electrolyte. The reduction of DO is proportional to BOD concentration. Ten minutes later, the optical and electrochemical signals are recorded. Then, the PBS solution with saturated DO was added and measured under the same conditions. The differences between the signals of the BOD standard solution and the PBS solution are the responses, and then we can accomplish the BOD quantitative detection curve.

PAn-FeTSPP film is grown on the gold substrate from $0.5 \mathrm{M}$ $\mathrm{H}_{2} \mathrm{SO}_{4}$ containing $0.1 \mathrm{M}$ aniline and $0.05 \mathrm{mM}$ FeTSPP by electrosynthesis. ${ }^{16}$ The process to BOD detection is similar to that of PAn-modified electrode. The difference is that a cathodic current of $-2.5 \mu \mathrm{A}$ is applied to the modified electrode.

\section{RESULTS AND DISCUSSION}

\section{A. BOD detection with the PAn-modified EC-TIRIE sensor}

A PAn film is generated on a gold chip by the CV method. According to Faraday's law of electrolysis, ${ }^{18}$ the estimated thickness of the PAn film on the gold chip is $118 \mathrm{~nm}$ (see the supplementary material $^{23}$ for obtaining). AFM images (see supplementary material ${ }^{23}$ ) show that the PAn film has a rough surface. After PAn film generation, the modified gold chip is fabricated as shown in Fig. 1 for BOD measurement. Figure 3 shows the real-time electrochemical and optical signals of the PAn modified EC-TIRIE sensor to solutions with different BOD values. As shown in Fig. 3(a), the potential increases gradually with the decrease in BOD concentrations. This is because the lower the BOD value, the less DO is consumed by the micro-organisms to degrade the organic matter, and the more DO is diffused into the electrolyte through the oxygen permeable membrane. In order to obtain the difference in DO concentration
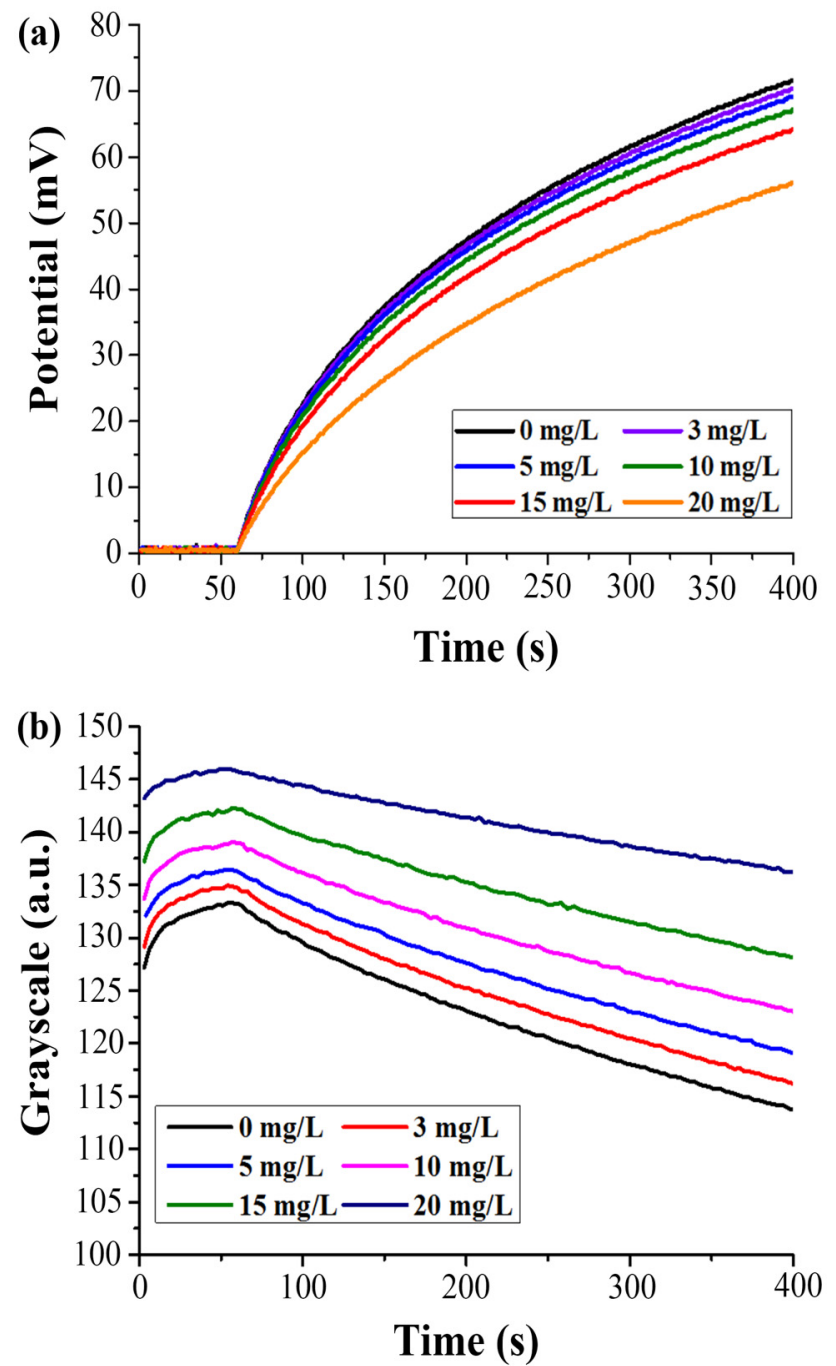

FIG. 3. Real-time electrochemical (a) and optical (b) responses of the PAn-modified EC-TIRIE sensor to solutions with different BOD values.

before and after degradation, we need to set the signal of 0-BOD solution as the background signal. And the responses of the sensor are the signal differences between BOD standard solutions and the $0-B O D$ solution. The BOD quantitative detection curve obtained by this method is shown in Fig. 4; within the range of 3-20 mg $\mathrm{l}^{-1}$ of BOD concentrations, both optical and electrochemical signals show linear with BOD concentrations. And the sensitivities of the optical response and the electrochemical response to BOD detection are 0.91 gray scale $\mathrm{mg}^{-1} \mathrm{l}$ and $1.06 \mathrm{mV} \mathrm{mg}^{-1} 1$, respectively.

In order to compare BOD detection of the PAn-modified EC-TIRIE sensor with a commercial BOD biosensor, standard solutions of different BOD values including $3,5,7,10$, and $15 \mathrm{mgl}^{-1}$ were prepared and detected by these two methods. The results are shown in Fig. 5; compared with the commercial BOD biosensor, the 


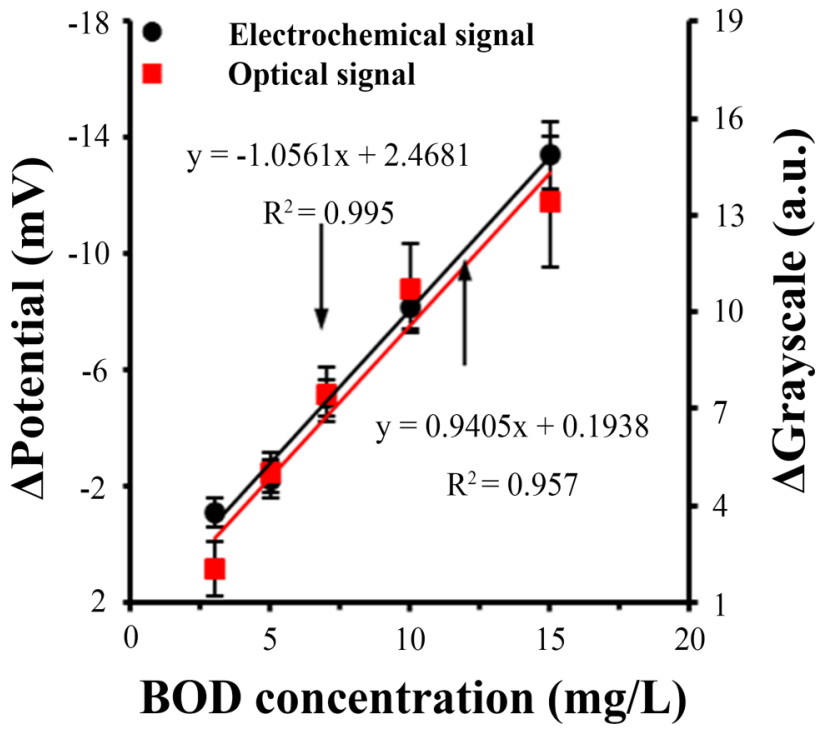

FIG. 4. BOD quantitative detection curves of the PAn-modified EC-TIRIE sensor.

deviation of both optical and electrochemical signals of the PAn-modified EC-TIRIE sensor is less than $15 \%$, which indicates that these two methods have good correlation to BOD detection.

\section{B. BOD detection using PAn-FeTSPP-modified EC-TIRIE sensor}

In order to improve the response of the PAn modified electrode to BOD detection, an oxygen-sensitive molecule, FeTSPP

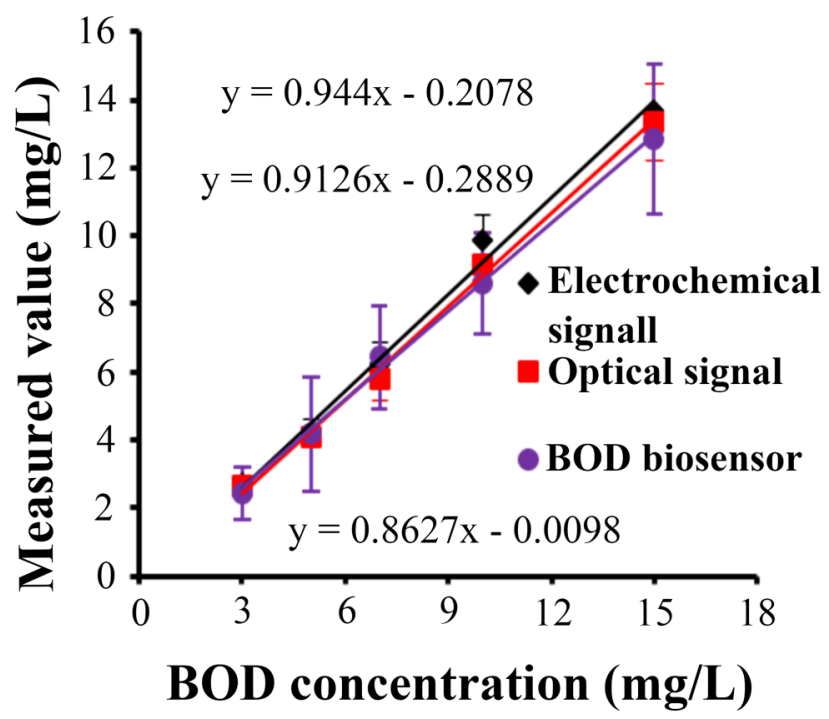

FIG. 5. Comparison of BOD detection by PAn-modified EC-TIRIE Sensor and the commercial BOD biosensor. is incorporated into PAn during the film generation. To detect and record the real-time response curves of standard solutions with different BOD values, the similar method to the PAn-modified electrode is used. Both the electrochemical and optical real-time signals (shown in Fig. 6) of PAn-FeTSPP-modified electrode to BOD detection are similar to PAn-modified electrode. The difference is that the variations of the two signals have been increased.

As seen in Fig. 7, incorporation of FeTSPP has improved the sensitivity of the PAn-modified electrode for BOD detection; the sensitivities of the optical response and the electrochemical responses have been improved to 1.22 gray scale $\mathrm{mg}^{-1} 1$ and $2.21 \mathrm{mV} \mathrm{mg}^{-1} \mathrm{l}$, respectively. And the linear relationship between the PAn-FeTSPPmodified electrode and the concentrations of BOD is better.

Since PAn is an oxygen-sensitive polymer, with reaction to DO, its "doping level" changes with the redox state remarkably, which results in the variation of both the dielectric constant and the

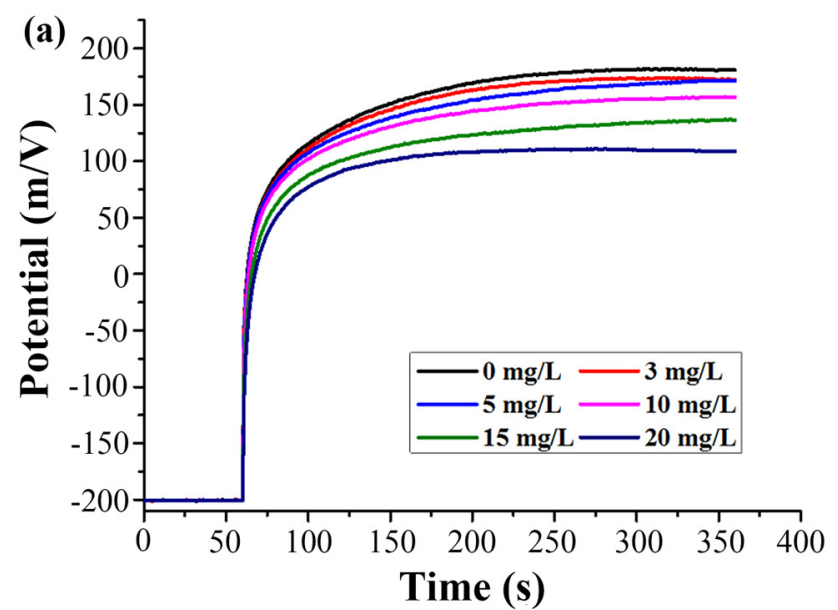

(b)

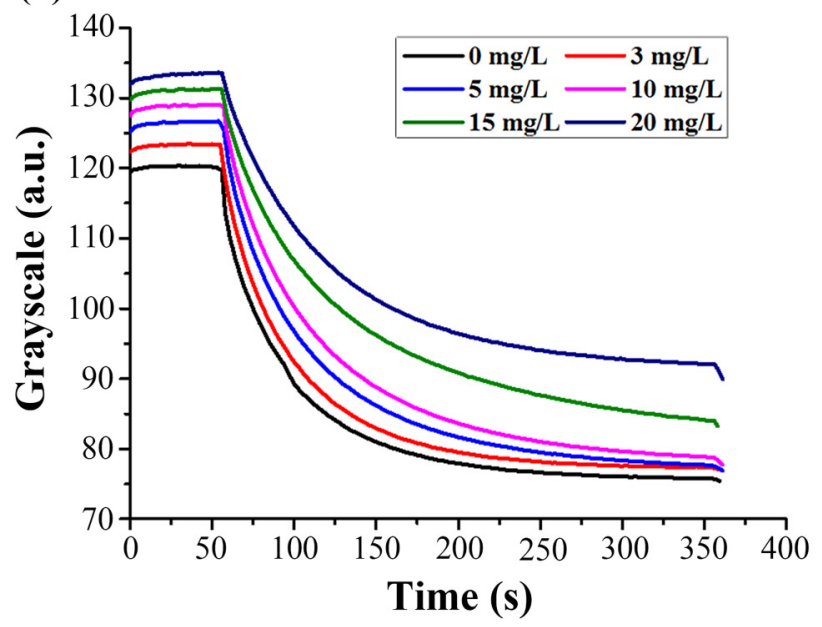

FIG. 6. Real-time electrochemical (a) and optical (b) responses of the PAn-FeTSPP-modified EC-TIRIE sensor to solutions with different BOD values. 


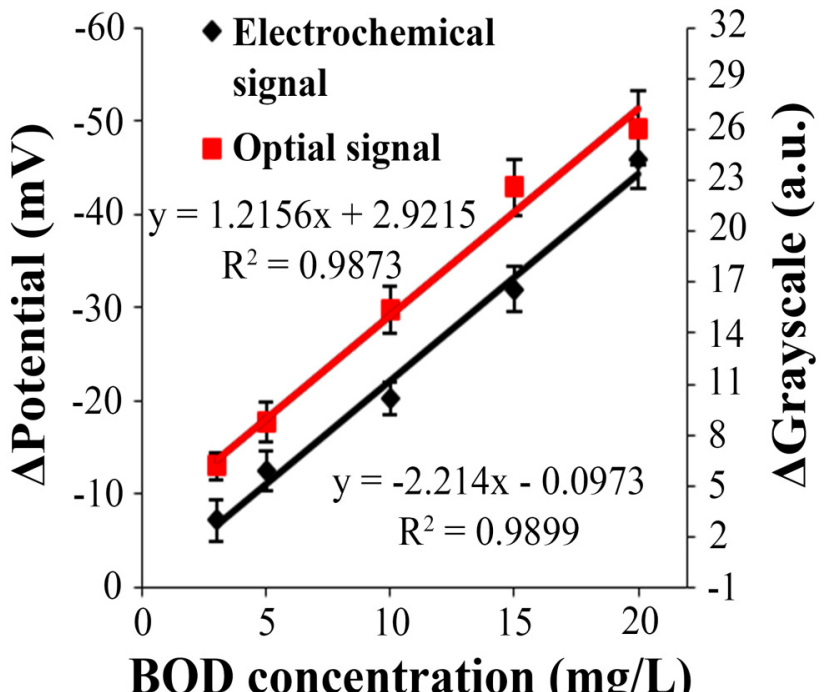

FIG. 7. BOD quantitative detection curves of the PAn-FeTSPP-modified EC-TIRIE sensor.

thickness. In order to investigate the relationship between the optical and electrochemical responses to DO, a glass/Au/PAn-based film/ liquid model has been established. We will discuss the influence factors of the optical response and the electrochemical response.

\section{Optical response}

For TIRIE using conventional polarizer-compensator-sampleanalyzer (PCSA) configuration, the fractional change $\delta I / I$ of the optical response $\delta I$ is given by (see the supplementary material ${ }^{23}$ for deduction)

$$
\frac{\delta I}{I}=\frac{\delta \boldsymbol{R}_{\boldsymbol{p}}}{\boldsymbol{R}_{\boldsymbol{p}}}+\alpha_{1} \delta \psi+\alpha_{2} \delta \Delta,
$$

where $\boldsymbol{R}_{\boldsymbol{p}}$ is the reflectance of the surface p-polarized light, given by

$$
\boldsymbol{R}_{\boldsymbol{p}}=\left|R_{p}\right|^{2} .
$$

$\alpha_{1}$ and $\alpha_{2}$ are the ellipsometric parameter coefficients and can be given by

$$
\begin{gathered}
\alpha_{1}=\frac{2[\tan \bar{\psi}(1+\cos 2 A)-\sin 2 A \sin (2 P+\bar{\Delta})]}{[1-\cos 2 \bar{\psi} \cos 2 A+\sin 2 \bar{\psi} \sin (2 P+\bar{\Delta}) \sin 2 A]}-\frac{4}{\sin 2 \psi}, \\
\alpha_{2}=-\frac{\sin 2 \bar{\psi} \cos (2 P+\bar{\Delta}) \sin 2 A}{[1-\cos 2 \bar{\psi} \cos 2 A+\sin 2 \bar{\psi} \sin (2 P+\bar{\Delta}) \sin 2 A]},
\end{gathered}
$$

where $\bar{\psi}$ and $\bar{\Delta}$ are the unperturbed values of the ellipsometric parameters $\psi$ and $\Delta ; \mathrm{P}$ and $\mathrm{A}$ are the polarizer and analyzer azimuths, respectively.

Equation (1) implies that the optical signal can be divided into two parts: the response from the SPR and that from the reflection polarization modulation of the surface. In fact, Nabok had proved that the sensitivity of the TIRIE is more than 30 times larger than that of the conventional SPR, ${ }^{19}$ which means that the polarization modulation rather than SPR dominates the signal response. On the other hand, Kyle J. Foley et al. have discussed the influence of the potential variation on SPR response, and they have proven that SPR response is also proportional to the potential disturbance. ${ }^{20,21}$ Besides, in Eq. (1), $\alpha_{1}$ and $\alpha_{2}$ are all constant in a certain experiment. Thus, we will put emphasis on the influence of the redox state of PAn on the ellipsometric parameters $\rho\left(\rho=\frac{R_{p}}{R_{s}}=\tan \psi e^{i \Delta}\right)$.

According to the supplementary material, ${ }^{23}$ the influence of the thickness and the dielectric constant of the PAn on the ellipsometric parameters $\rho$ is given by the following equation:

$$
\frac{\delta \rho}{\rho}=\kappa_{2} \delta \varepsilon_{2}+\kappa_{4} \delta d_{2} .
$$

On the other hand, we have

$$
\rho=\tan \psi e^{i \Delta} .
$$

And then, by taking logarithmic differential of Eq. (6), we obtain

$$
\frac{\delta \rho}{\rho}=\frac{2}{\sin 2 \psi} \delta \psi+j \delta \Delta
$$

Therefore,

$$
\begin{gathered}
\delta \psi=\left(\frac{1}{2} \sin 2 \psi\right) \operatorname{Re}\left(\frac{\delta \rho}{\rho}\right), \\
\delta \Delta=\operatorname{Im}\left(\frac{\delta \rho}{\rho}\right) .
\end{gathered}
$$

Taking Eq. (5) into Eqs. (8) and (9), we have

$$
\begin{aligned}
\delta \psi & =\left(\frac{1}{2} \sin 2 \psi\right) \operatorname{Re}\left(\frac{\delta \rho}{\rho}\right) \\
& =\left(\frac{1}{2} \sin 2 \psi\right) \operatorname{Re}\left(\kappa_{2} \delta \varepsilon_{2}+\kappa_{4} \delta d_{2}\right), \\
\delta \Delta & =\operatorname{Im}\left(\frac{\delta \rho}{\rho}\right)=\operatorname{Im}\left(\kappa_{2} \delta \varepsilon_{2}+\kappa_{4} \delta d_{2}\right) .
\end{aligned}
$$

\section{Electrochemical response}

According to the Nernst equation, we have

$$
\varphi=\varphi^{\theta}+\frac{\mathrm{RT}}{n \mathrm{~F}} \ln \left(\frac{f}{1-f}\right),
$$

where $\varphi^{\theta}$ is the standard electrode potential, $\mathrm{R}$ is the universal gas constant, $\mathrm{T}$ is the temperature in kelvin, $\mathrm{F}$ is the Faraday constant, and $n$ is the number of electron transfers in the electrode reaction; as for $f$, we suppose it is the proportion of the area of oxidation to 
the total area of PAn. Because some portion is exposed to oxygen earlier than other portion, the oxidation state of PAn is not evenly distributed.

The dielectric constant and the thickness of PAn are different in the oxidation state and the reduction state. ${ }^{22}$ We have assumed that

$$
\begin{aligned}
& \varepsilon_{2}=f \varepsilon_{\mathrm{o}}+(1-f) \varepsilon_{\mathrm{r}}, \\
& d_{2}=f d_{\mathrm{o}}+(1-f) d_{\mathrm{r}},
\end{aligned}
$$

where $\varepsilon_{\mathrm{o}}, \varepsilon_{\mathrm{r}}, d_{\mathrm{o}}$, and $d_{\mathrm{r}}$ represent the dielectric constant of the oxidation state and the reduction state of PAn, and the thickness of the oxidation state and the reduction state of PAn, respectively.

According to the supplementary material, ${ }^{23}$ we can conclude that the electrode potential variation of the PAn-modified surface, $\delta \psi$, the dielectric constant variation of the PAn film, $\delta \varepsilon_{2}$, and the thickness variation of the PAn film, $\delta d_{2}$, are all proportional to the ratio change of the area of the oxidized part of PAn, $\delta f$. That is to say

$$
\begin{gathered}
\delta \varphi \propto \delta f \\
\delta \varepsilon_{2} \propto \delta f, \quad \delta d_{2} \propto \delta f .
\end{gathered}
$$

\section{E. Relationship between the optical response and the electrochemical response}

According to Eqs. (17), (11), and (12), we have

$$
\delta \psi \propto \delta f, \quad \delta \Delta \propto \delta f .
$$

Also, we already know that the SPR response is proportional to potential disturbance; according to Eqs. (15) and (17), we know

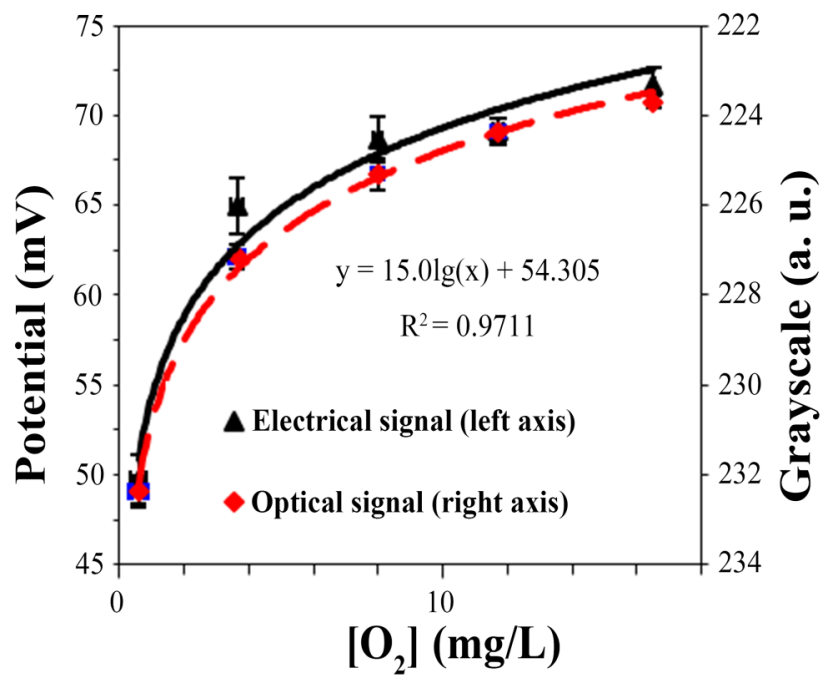

FIG. 8. Responses in both electrochemical (black solid line) and optical (red dashed line) signals by EC-TIRIE sensor with PAn-modified electrode upon different oxygen concentrations (error bars represent three repeated experiments). that $\delta \varphi$ and $\delta \Delta$ are all proportional to $\delta \varphi$. Therefore, according to Eq. (1), the relationship between optical signal and electrochemical signal is given by

$$
\delta I \propto \delta \varphi
$$

Our results have shown the relationship between the electrochemical and optical signals of the EC-TIRIE sensor with the PAn-modified electrode. Figure 8 shows that with the PAn-modified electrode, both the electrochemical and optical signals to the DO concentrations show a logarithmic correlation, which is similar to the Nernst equation. It can also be observed clearly that the optical signal variation tends to keep pace with the electrochemical signal, which indicates that the change of optical signal is proportional to the electrochemical signal, and also confirms that our analysis is correct.

\section{CONCLUSION}

In this paper, we use an EC-TIRIE sensor that combines electrochemical and optical systems to detect BOD by immobilizing a microbial membrane. The gold chip is modified with PAn and PAn containing FeTSPP, respectively, to improve the sensitivity of the sensor to BOD detection. The results show that the combined sensor is effective for BOD detection, both the optical and the electrochemical signals have a linear relationship with BOD concentrations, and incorporation of FeTSPP has improved both the optical and electrochemical signals of PAn to BOD. Finally, the relationship between the optical and electrochemical responses to DO was discussed, which agrees well with experimental results. Since BOD detects the difference in DO before and after microbial degradation, multiple channels can be used in future studies, which can greatly improve the detection efficiency.

\section{ACKNOWLEDGMENTS}

The authors thank the financial support to the Youth Innovation Promotion Association of the Chinese Academy of Sciences (Grant No. 2017026), the National Natural Science Foundation of China (NNSFC) (Grant No. 81872584), the National Basic Research Program of China (Grant No. 2015CB352100), and the International Science and Technology Cooperation Program of China (Grant No. 2015DFG32390).

\section{REFERENCES}

${ }^{1}$ C. Liu, C. Ma, D. Yu, J. Jia, L. Liu, B. Zhang, and S. Dong, Biosens. Bioelectron. 26, 2074 (2011).

${ }^{2}$ N. Yoshida, J. Hoashi, T. Morita, S. J. McNiven, H. Nakamura, and I. Karube, J. Biotechnol. 88, 269 (2001).

${ }^{3}$ I. Karube, T. Matsunaga, S. Mitsuda, and S. Suzuki, Biotechnol. Bioeng. 19, 1535 (1977).

${ }^{4}$ I. Chang, J. Jang, G. C. Gil, M. Kim, H. J. Kim, B. W. Cho, and B. H. Kim, Biosens. Bioelectron. 19, 607 (2004).

${ }^{5}$ M. N. Kim and H. S. Kwon, Biosens. Bioelectron. 14, 1 (1999).

6. Liu and B. Mattiasson, Water Res. 36, 3786 (2002).

${ }^{7}$ G. J. Chee, Y. Nomura, K. Ikebukuro, and I. Karube, Biosens. Bioelectron. 15, 371 (2000)

${ }^{8}$ L. Nei and R. G. Compton, Sens. Actuators B 30, 83 (1996). 
${ }^{9} \mathrm{M}$. Li, W. Liu, J. P. Correia, A. C. Mourato, A. S. Viana, and G. Jin, Electroanalysis 26, 374 (2014).

${ }^{10}$ J. Kankare and I. A. Vinokurov, Anal. Chem. 69, 2337 (1997).

${ }^{11}$ B. Batra, S. Kumari, and C. S. Pundir, Enzyme Microb. Technol. 57, 69 (2014).

${ }^{12}$ R. Rawal, S. Chawla, and C. S. Pundir, Anal. Biochem. 419, 196 (2011).

${ }^{13}$ D. D. Sawall, R. M. Villahermosa, R. A. Lipeles, and A. R. Hopkins, Chem. Mater. 16, 1606 (2004).

${ }^{14}$ C. S. Kushwaha and S. K. Shukla, J. Mater. Sci. 54, 10846 (2019).

${ }^{15} \mathrm{X}$. Hun, Y. Li, S. Wang, Y. Li, J. Zhao, H. Zhang, and X. Luo, Biosens. Bioelectron. 112, 93 (2018).

${ }^{16}$ M. Li, I. M. Ornelas, W. Liu, Y. Niu, J. P. Correia, A. S. Viana, and G. Jin, Electroanalysis 27, 1427 (2015).
${ }^{17}$ L. Liu, Y. Chen, Y. Meng, S. Chen, and G. Jin, Thin Solid Films 519, 2758 (2011).

${ }^{18}$ N. Plesu, A. Kellenberger, M. Mihali, and N. Vaszilcsin, J. Non-Cryst. Solids 356, 1081 (2010).

${ }^{19}$ A. V. Nabok, A. Tsargorodskaya, A. K. Hassan, and N. F. Starodub, Appl. Surf. Sci. 246, 3816 (2005).

${ }^{20}$ X. Shan, K. J. Foley, and N. Tao, Appl. Phys. Lett. 92, 133901 (2008).

${ }^{21}$ W. Liu, Y. Niu, A. S. Viana, J. P. Correia, and G. Jin, Anal. Chem. 88, 3211 (2016).

${ }^{22}$ Z. Wang et al., Anal. Chem. 91, 6769 (2019).

${ }^{23}$ See supplementary material at https://doi.org/10.1116/1.5129606 for the EC-TIRIE system response. 